\title{
Helicobacter pylori detection methods in complex samples: a mini-review
}

\begin{abstract}
Helicobacter Pylori (HP) bacteria is considered a very dangerous pathogen with about half the earth population are host. It infects the human gastrointestinal tract causing permanent damages that may lead to cancer. The exact routes of Helicobacter pylori infection transmission is still not fully investigated. Thus, the in-vitro detection of HP is very important in monitoring infections at its early stages and in contaminated mediums like food and water. Literature reports several techniques and approaches to detect HP in-vitro, but mostly with the cost of complex instruments, excessive sample processing and time consuming test methods making them not suitable for affordable routine procedure. This paper contributes in briefly reviewing the few recent detection methods of HP in-vitro. Experience of authors in HP in-vitro detection is presented. The influence of the detection method on sensitivity, selectivity and on compatibility with point-of-care devices is overviewed. Major types of sensors based on antigen-antibody interaction or DNA hybridization are discussed.
\end{abstract}

Volume 5 Issue 5 - 2019

\author{
Hussamaldeen Jaradat,' Mohammed Ibbini \\ 'Department of Information Technology, Jordan University of \\ Science and Technology, Jordan \\ ${ }^{2}$ Department of Biomedical Engineering, Jordan University of \\ Science and Technology, Jordan \\ Correspondence: Mohammed Ibbini, Department of \\ Biomedical Engineering, Jordan University of Science and \\ Technology, Jordan, Tel 00962795597908 \\ Emailmohib@just.edu.jo
}

Received: October 03, 2019 | Published: October 22, 2019

Keywords: helicobacter pylori, sensors, complex sample, nanotechnology

\section{Introduction}

HP is gram-negative fastidious and pathogenic bacteria of high geographical widespread with almost half of world's population is a host. ${ }^{1,2}$ It is considered to be the main cause of gastrointestinal cancers in human ${ }^{1,2} \mathrm{HP}$ attacks stomach epithelial cells by injecting its virulent factors. ${ }^{3-5} \mathrm{HP}$ infection can occur through human interaction with other host or with contaminated stool, water and food. ${ }^{6,7} \mathrm{Up}$ to date, the exact routes of HP infection transmission is still not fully investigated. ${ }^{7} \mathrm{HP}$ is showing an increasing resistance for the routine medical treatments by antibiotics. ${ }^{3,10-13}$ Thus, HP contamination detection is considerably important to prevent infections or at least diagnose the infection at early stages rather than just after serious disease and symptoms development. HP can cause several diseases, but not all hosts will suffer from symptoms as not all HP strains are seriously pathogenic., ${ }^{3,10-13}$ In addition, disease development also depends on host conditions like lifestyle and diet. ${ }^{3,10-13}$ Thus, the main challenge in HP treatment is to diagnose infection at early stage especially for hosts most likely to develop serious HP-related diseases and in host children. ${ }^{10}$ Additionally, diagnosing HP medium contaminations within its susceptible transition routes like food, water and communal facilities will be very important to prevent infections. Since the discovery of HP, several techniques have been proposed to detect infection of HP in human both of invasive and non-invasive nature. ${ }^{11}$ Invasive methods, like histopathology staining and routine endoscopy, are typically oriented for detection in infected humans especially at late stages. ${ }^{3,10-13}$ However, non-invasive detection methods can be of in-vivo and in-vitro nature., $3,7,10,11$ Urea Breath Test (UBT) is very common and widely used as in-vivo detection method of HP. ${ }^{3,10-13}$ Colorimetric sensors, Fluorescent-based sensors, Electrochemical sensors, ELISA kits as well as Lateral Flow Devices provide In-vitro detection of HP. ${ }^{14}$ Nevertheless, there is still a lack of cost-effective, ultrasensitive, specific and rapid sensors to diagnose HP contamination in complex samples while being compatible with point-of-care devices. This is particularly important in preventive diagnosis of food/water supplies as well as diagnosis of infection at early stages. A brief overview of the recently developed HP detection in-vitro is presented in this mini-review. In addition, it discusses the advantages and disadvantages of the proposed methods and outline possible future directions in this issue.

\section{Discussion}

\section{HP infection diagnosis}

HP diagnosis is very useful at early stage infection as in childhood and before developing symptoms. Many methods have been proposed and developed to detect HP with varying reliabilities in terms of sensitivity and accuracy. ${ }^{5-10}$ Invasive and non-invasive tests have been proposed based on different properties and pathogenic behaviors of HP. ${ }^{5-10}$ These tests have different factors that determine its suitability for use as HP diagnosis. ${ }^{11}$ These factors can range from the host clinical status, cost, safety as well as sample preparation., ${ }^{3,40-13}$ Invasive methods are normally used in patients with symptoms and beyond the discussion of this mini-review. Non-invasive methods are particularly important for it is general simplicity, minimal effect on patient, cost-effectiveness, minimal sample preparation and their potential for point-of-care devices. ${ }^{4,11-13}$ UBT is very famous method for non-invasive in-vivo HP infection using $\mathrm{C}^{14}$ labeled urea, that relies on measuring $\mathrm{CO}_{2}$ production from $\mathrm{HP}$ in the stomach as an evidence for its activity. ${ }^{14}$ However, this test is not very suitable for early stage detection and for detection in children because of low production of $\mathrm{CO}_{2} \cdot{ }^{10,14} \mathrm{In}$ addition, it can be only applied in-vivo and requires infrastructure. In-vitro methods mainly rely on utilizing different HP biomarkers like antigens and outer membrane proteins (OMP) or DNA-based markers to detect the existence of HP. ${ }^{3,10-13}$ One of the famous non-invasive in-vitro methods is the HP stool antigen (HpSA) method. ${ }^{11,15}$ This antigen is produced by the human body as a response of HP infection. . $^{5-17,14} \mathrm{HpSA}$ can be found in blood serum, stool and saliva and can be tested using the conventional serological technique like enzyme-linked immunosorbent Assay (ELISA)., ${ }^{3,10,11}$ This test is considered to be simple enough and compatible with point 
of care devices but increasing number of studies start to question its reliability as it produces more false-negative results. ${ }^{10,11}$

\section{Fluorescent detection methods}

Fluorescent techniques are widely used in different fields of science due to characteristics including inherent sensitivity, ease of handling and the availability of several dyes making them promising for sensing applications. ${ }^{13,16}$ Semiconductor Quantum Dots (QD's) based fluorescence dyes can be conjugated with biomolecules to produce sensing probes. ${ }^{15} \mathrm{CuInS} 2$ QDs covalently labeled with single stranded DNA (ssDNA) complementary for HP DNA were prepared by Liu et al. ${ }^{16}$ It showed great sensitivity and selectivity of HP even in complex sample. ${ }^{16}$ Another study carried out by Hong et. al. 2019 showed the feasibility of using the $\mathrm{pH}$-sensitive benzothiazole conjugated with hydroxythiophene $\left(\mathrm{T}_{2}(\mathrm{OH}) \mathrm{B}\right)$ as fluorescence probe. ${ }^{13}$ Chen et al. ${ }^{10}$ proposed a novel method of using immunomagnetic beads (IMP's) attached to antibody-conjugating QD probe that can be effectively used in complex sample. ${ }^{10}$ They have functionalized monoclonal antibodies (mAbs) with IMP to capture and concentrate HP from the complex sample, then the complex was eluted and allowed to interact with polyclonal antibodies (pAbs) functionalized with fluorescence QD's. ${ }^{10}$ Fluorescence-based HP detection still need the use of bulky instruments or smartphones loaded with proper toolkit especially for precise quantitative measurements. This specifically limits the opportunity for effective use in quantitative measurements in remote regions. In addition, it requires relatively long procedure time. ${ }^{13,16,17}$

\section{Colorimetric methods}

Colorimetric sensors basically use the change of the reflected or absorbed intensity of light on biological labeled-reagent complex structure upon interaction with the target. ${ }^{17,18}$ This shift in optical properties is originated from the structural changes or the Surface Plasmon Resonance (SPR) of the complex..$^{17,18}$ Ali et al. ${ }^{3}$ proposed a colorimetric sensor based on the DHp3T4 shortened cleavage DNAzyme tagged with urease and immobilized by agarose beads. ${ }^{3}$ In their work, adding the sample containing HP to the sensor will result in freeing urease which will be collected by centrifugation then adding it into phenol-red and urea solution. ${ }^{7}$ As a result, urease will hydrolyze urea releasing ammonia resulting in raising $\mathrm{pH}$ and consequently changing the color from yellow to red. ${ }^{3}$ This methodology resulted in limit of detection as low as $10^{4} \mathrm{cfumL}^{-1} \mathrm{HP}$ in stool sample. ${ }^{3}$ They also demonstrated a paper-based sensor version resulting in half quantitative measurements and a sensor shelf life of around 4 months as reported by author. ${ }^{7}$ Colorimetric sensors use color tone changes to quantify interactions, thus it should be always utilized with colorsensitive optical devices and enabling software. Point-of-care device could be successful upon the effective utilization of smartphones for their well-known highly-sensitive cameras and computational power. Qualitative result readout of colorimetric sensors still can be done visually.

\section{Electrochemical methods}

Electrochemical sensors have inherently better sensitivity, reproducibility and portability than other sensing methods. ${ }^{8,19}$ Standard electrochemical sensor utilizes the well-know 3-electrode configuration: Working Electrode (WE), Counter Electrode (CE) and Reference Electrode (RE). WE and CE are surface-functionalized using the same nanomaterials for signal amplification. ${ }^{17,19,20} \mathrm{We}$ is further surface modified to specifically bind with HP biomarker usually through ssDNA hybridization or antigen-antibody interactions..$^{17,19}$ The electrical circuit path for injecting actuating signal and perform the measurement is completed by using redox-active electrolyte solution that contains the HP biomarker. Typical electrochemical analysis tests are Cyclic Voltammetry (CV), Differential Pulse Voltammetry (DPV) and Electrochemical Impedance Spectroscopy (EIS). ${ }^{12,17,19}$ Peng et al. ${ }^{12} 2017$ designed HP gold electroactive electrode labeled with $\beta$-cyclodextrin ( $\beta$-CD) DNA as biosensor. ${ }^{8}$ DPV technique were used to measure HP on the designed sensor that showed lower limit of detection as low as $0.15 \mathrm{nM} .{ }^{12}$ Electrochemical based sensing requires the use of potentiostat devices to perform electrical supply and signal analysis. On the other hand, electrochemical sensors can be fabricated using screen-printed electrode technology with completely disposable materials like paper and still produce fully functional measurements. ${ }^{21}$

\section{Conclusion}

HP detection in early stages and in contaminated medium that human may interact with is very important to control this dangerous pathogen. Many sensor approaches and techniques have been proposed to detect the HP infection and contamination in-vitro. Optical sensing approaches like, fluorescence and colorimetric, exhibit some attractive characteristics as high sensitivity and selectivity. Yet, these methods normally require optical analysis and further image processing techniques and still suffer from elongated test time. Electrochemical sensing provides higher sensitive electrical measurements and is promising for cost-effective point-of care-devices use, due to their simple and affordable design, especially using paper-based screen printed electrodes technology. Consequently, a cost effective, easy detection of HP in a complex samples remains a major demand. Therefore, current research trend is to develop a cheap, point-of-care compatible, fast and sensitive detection of HP in samples of complex nature like stool, drinking water, food and human saliva ...etc. Possible enhancements may range from use of newly discovered HP OMP's biomarkers along with paper-based screen printed electrode technology. Moreover, developing generic, portable and affordable electronic point-of-care device similar to that used in glucose analysis will greatly help in achieving portability.

\section{Funding details}

None.

\section{Acknowledgements}

None.

\section{Conflicts of interest}

Authors declare that there is no conflict of interest.

\section{References}

1. Salama NR, Hartung ML, Müller A. Life in the human stomach: persistence strategies of the bacterial pathogen Helicobacter pylori. Nature Reviews Microbiology. 2013;11(6):385-399.

2. Königer V, Holsten L, Harrison et al. Helicobacter pylori exploits human CEACAMs via HopQ for adherence and translocation of CagA. Nature Microbiology. 2016;2(1).

3. Ali MM, Wolfe M, Tram K, et al. A DNAzyme-Based Colorimetric Paper Sensor for Helicobacter pylori. Angewandte Chemie International Edition. 2019;58(29):9907-9911. 
4. Uemura N, Okamoto S, Yamamoto S, et al. Helicobacter pyloriInfection and the Development of Gastric Cancer. New England Journal of Medicine. 2001;345(11):784-789.

5. Khatoon J. Role of Helicobacter pylori in gastric cancer: Updates. World Journal of Gastrointestinal Oncology. 2016;8(2):147-158.

6. Chauhan N, Gupta S, Avasthi DK, et al. Zinc Oxide Tetrapods Based Biohybrid Interface for Voltammetric Sensing of Helicobacter pylori. ACS Applied Materials \& Interfaces. 2018;10(36):30631-30639.

7. $\mathrm{Ng} \mathrm{CG}$, Loke MF, Goh KL, et al. Biofilm formation enhances Helicobacter pylori survivability in vegetables. Food Microbiology. 2017;62:68-76.

8. Talebi Bezmin Abadi A. Helicobacter pylori: Emergence of a Superbug. Frontiers in Medicine. 2014:1.

9. Ghotaslou R. Prevalence of antibiotic resistance in Helicobacter pylori: A recent literature review. World Journal of Methodology. 2015;5(3):164.

10. Chen L, Li X, Zou T, et al. Ultrasensitive detection of H. pylori in human feces based on immunomagnetic bead capture and fluorescent quantum dots. The Analyst. 2019;144(13):4086-4092.

11. Khalilpour A, Kazemzadeh-Narbat M, Tamayol A, et al. Biomarkers and diagnostic tools for detection of Helicobacter pylori. Applied Microbiology and Biotechnology. 2016;100(11):4723-4734

12. Peng P. Fabrication of an Electrochemical Sensor for Helicobacter pylori in Excrement Based on a Gold Electrode. International Journal of Electrochemical Science. 2017:9478-9487.

13. Hong KI, Park SH, Lee SM, et al. A pH-sensitive excited state intramolecular proton transfer fluorescent probe for imaging mitochondria and Helicobacter pylori. Sensors and Actuators B: Chemical. 2019;286:148-153.
14. Best LM, Takwoingi Y, Siddique S, et al. Non-invasive diagnostic tests for Helicobacter pylori infection. Cochrane Database of Systematic Reviews. 2018.

15. Shimoyama T. Stool antigen tests for the management of Helicobacter pylori infection. World Journal of Gastroenterology. 2013;19(45):8188.

16. Liu Z, Su X. A novel fluorescent DNA sensor for ultrasensitive detection of Helicobacter pylori. Biosensors and Bioelectronics. 2017;87:66-72.

17. Zarei M. Portable biosensing devices for point-of-care diagnostics: Recent developments and applications. TrAC Trends in Analytical Chemistry. 2017;91:26-41.

18. Vilela D, González MC, Escarpa A. Sensing colorimetric approaches based on gold and silver nanoparticles aggregation: Chemical creativity behind the assay. A review. Analytica Chimica Acta. 2012;751:24-43.

19. Kaushik A, Yndart A, Kumar S, et al. A sensitive electrochemical immunosensor for label-free detection of Zika-virus protein. Scientific Reports. 2018;8(1).

20. Maduraiveeran G, Sasidharan M, Ganesan V. Electrochemical sensor and biosensor platforms based on advanced nanomaterials for biological and biomedical applications. Biosensors and Bioelectronics. 2018;103:113129.

21. Smith S, Madzivhandila P, Ntuli L, et al. Printed Paper-Based Electrochemical Sensors for Low-Cost Point-of-Need Applications. Electrocatalysis. 2019;10(4):342-351. 